\title{
Prevalencia de consumo de alcohol en estudiantes de la Facultad de Medicina en la Universidad Católica del Maule
}

\author{
MARÍA LATORRES ${ }^{a}$, ANDREA HUIDOBRO
}

Departamento Ciencias

Preclínicas Facultad de

Medicina Universidad

Católica del Maule.

${ }^{a}$ Estudiante de Medicina

Universidad Católica del

Maule.

Recibido el 24 de octubre de 2011, aceptado el 29 de mayo de 2012

Correspondencia a:

Dra. María Nelly Latorres

Facultad de Medicina

Universidad Católica del

Maule

Avda. San Miguel 3605

Casilla 617

Talca, Chile.

Fax: 005671203435

E-mail:mnelly89@gmail.

\section{Prevalence of alcohol consumption among medical students at the Universidad Católica del Maule, Chile}

Background: Teenagers and university students are an especially vulnerable population towards unhealthy alcohol consumption Aim: To assess the actual consumption of alcohol among medical students at the Universidad Católica del Maule, Chile (UCM). Material and Methods: A cross sectional study of students who entered medical school between years 2004 and 2010. We applied CAGE questionnaire along with questions about frequency of alcohol consumption. Results: From a universe of 317 students, 216 (age range 17-27 years, 54\% females) answered the survey (68.8\%), $64.2 \%$ admitted to consume alcohol and $6 \%$ had alcohol dependence. Seventy three percent of the alcohol drinkers were males. Conclusions: The prevalence of alcohol consumption in medical students of the UCM is significantly lower than the prevalence in students of another University surveyed in 2005 (74\%) and it is similar to the national statistics. Alcohol consumption was higher in men that in women.

(Rev Med Chile 2012; 140: 1140-1144).

Key words: Alcohol drinking; Questionnaires; Students, medical.
$\mathrm{E}$ 1 abuso en el consumo de alcohol es un problema importante de salud, tanto a nivel individual como colectivo. El consumo no moderado de estas sustancias contribuye al incremento de las muertes prematuras y a un aumento de la discapacidad en la población, ya sea como consecuencia de los padecimientos asociados al consumo (cirrosis hepática, cáncer de hígado, cáncer de esófago y dependencia alcohólica), como por los accidentes y violencias que se presentan bajo los efectos del alcohol ${ }^{1}$.

Los últimos estudios realizados por la OMS, estiman que $55 \%$ de la población general presenta algún nivel de consumo de alcohol, lo que estaría asociado a 2,5 millones de muertes al año ${ }^{2}$.

Según el Octavo Estudio Nacional de Drogas en población general de Chile 2008, realizado por el Consejo Nacional para el Control de Estupefacientes (CONACE), 60,8\% de los jóvenes entre 19 y 25 años de edad consume alcohol, por lo menos, una vez al mes. Si bien la cifra bajó con respecto a la medición anterior realizada en el año 2006, el rango etario entre 19 y 25 años tuvo una disminución menor de consumo de alcohol con respecto al resto de la población ${ }^{3}$.

Los estudiantes de medicina no están al margen de estos indicadores. Existe bibliografía internacional que muestra que el consumo de alcohol tiende a ser relativamente alto en este grupo $^{4,5}$, lo que estaría relacionado fuertemente al estrés y presiones académicas propias de este grupo de estudiantes ${ }^{6}$. Sin embargo, en Chile, la bibliografía relacionada con el consumo de alcohol en estudiantes de medicina es muy escasa. Hasta donde los autores conocemos, sólo existe un estudio realizado a estudiantes de medicina de la Pontificia Universidad Católica de Chile (PUC), donde se encontró que $92 \%$ de los estudiantes 
ha consumido alcohol alguna vez en su vida, $89 \%$ ha consumido en el último año y $74 \%$, en el último mes ${ }^{7}$. Estos datos parecen preocupantes y contradictorios con la función que el médico debe ejercer en atención primaria, como líder de equipos de salud encargados de promover estilos de vida saludables, entre los cuales se encuentra el consumo moderado y no excesivo de alcohol.

Ante la falta de datos locales, creemos necesario conocer la magnitud del consumo de alcohol en estudiantes de medicina en provincia. Así el presente estudio se realizó en estudiantes de medicina de la Universidad Católica del Maule (VII región), con el fin de analizar y contrastar los resultados con la realidad general nacional del consumo de alcohol y con el estudio realizado en la Facultad de Medicina de la Pontificia Universidad Católica de Chile.

\section{Material y Método}

Se realizó un estudio de corte descriptivo en los estudiantes de medicina de ingreso 2004 a 2010 de la Universidad Católica del Maule, Talca, Chile.

Para ello se entregó un cuestionario a los estudiantes de Medicina de la Universidad Católica del Maule, por curso, desde ingreso 2004 a ingreso 2010 para ser autoadministrado. El cuestionario fue respondido luego de firmar consentimiento informado según los requerimientos del Comité de Ética de la Facultad de Medicina de la Universidad Católica del Maule.

Para medir el uso y el abuso del alcohol el cuestionario que se utilizó fue el CAGE, que corresponde a una encuesta que consta de cuatro preguntas cerradas, las que se centran en pensar en reducir el consumo de alcohol, la molestia por la crítica social sobre su consumo, el sentimiento de culpabilidad y la dependencia. Así, mientras más respuestas afirmativas, mayor es el grado de dependencia alcohólica. De esta forma, si se obtiene un puntaje de 0-1 se considera un "Bebedor social". Si se obtienen 2 puntos se considera un "consumo de riesgo". Si el puntaje es 3 representa un "consumo perjudicial" y, finalmente, si se obtiene 4 representará una "dependencia alcohólica" con una sensibilidad de $85 \%$ y especificidad de $90 \%{ }^{8}$.

Además, se midió la frecuencia de consumo de alcohol para poder clasificar a la población de acuerdo a su rango de consumo, preguntando al alumno, la cantidad de veces al mes que bebe y cuantas copas consume cuando lo hace.

Entre las variables medidas, la variable dependiente fue consumo de alcohol y las variables independientes fueron la edad, año de ingreso y sexo. Se midió el consumo de alcohol a través de la frecuencia mensual y la intensidad de consumo, la que se calculó en tragos o copas, donde una copa equivale a 13 gr de alcohol.

Se calculó el porcentaje de consumo en la población total y se analizó por género, edad y año de ingreso. Se analizó la asociación entre el consumo de alcohol y el puntaje CAGE con las variables sexo, edad, año de ingreso a través del programa estadístico STATA 9.0, utilizando Chi cuadrado para análisis de variable consumo de alcohol y género y test de " $\mathrm{T}$ " de Student para variables continuas. Por último, se analizó si existía diferencia entre los resultados obtenidos en el presente estudio y los de la Escuela de Medicina de la Pontificia Universidad Católica y de la Encuesta Nacional de Salud 2003.

\section{Resultados}

De un total de 317 estudiantes pertenecientes a la carrera de medicina de la Universidad Católica del Maule, 218 sujetos fueron encuestados $(68,8 \%)$. El promedio y desviación estándar de la edad de la muestra fue de 21,4 \pm 2 años (rango 17-27 años).

Del total de encuestados las mujeres representaron el 54,13\%, mientras que los hombres representaron $45,9 \%$ del total. Por otro lado, la muestra representó al $80 \%$ de las mujeres $(\mathrm{n}=118)$ y al $60 \%$ de los hombres $(\mathrm{n}=100)$.

De los encuestados 64,2\% afirmó beber alcohol, en tanto $35,8 \%$ se declaró abstemio. Del total de los hombres, $73 \%$ se declararon bebedores, mientras que $56,4 \%$ de las mujeres declararon beber alcohol $(p=0,011)$. No se encontró asociación significativa entre edad y consumo de alcohol $(\mathrm{p}=0,241)$.

La Tabla 1 muestra la frecuencia de bebedores por año de ingreso. La prevalencia de consumo de alcohol fue mayor en los estudiantes de internado, mientras que cuarto y quinto presentaronn las cifras más bajas de consumo, si bien esta diferencia no alcanzó a ser estadísticamente significativa $(\mathrm{p}=0,158)$. 
Tabla 1. Distribución de los alumnos de medicina de la Universidad Católica del Maule y prevalencia de consumo de alcohol según año de estudio

\begin{tabular}{|ccccc|}
\hline Año de estudio & $\begin{array}{c}\text { n de encuestados } \\
\text { (\% del total) }\end{array}$ & \% del curso & $\begin{array}{c}\text { n de } \\
\text { bebedores }\end{array}$ & \% de bebedores \\
\hline $1^{\circ}$ & $50(22,94)$ & 84,7 & 30 & 60,0 \\
\hline $2^{\circ}$ & $56(25,69)$ & 83,6 & 40 & 71,4 \\
\hline $3^{\circ}$ & $30(13,76)$ & 46,1 & 18 & 60,0 \\
\hline $4^{\circ}$ & $29(13.30)$ & 43,9 & 17 & 58,6 \\
\hline $5^{\circ}$ & $33(15,14)$ & 53,2 & 19 & 57,6 \\
\hline Internado & $20(9,17)$ & 26,0 & 16 & 80,0 \\
\hline Total & $218(100)$ & 68,3 & 140 & 64,2 \\
\hline
\end{tabular}

Tabla 2. Distribución de puntuación CAGE en los alumnos consumidores de alcohol de la Universidad Católica del Maule

\begin{tabular}{|ccc|}
\hline Puntaje CAGE* & Frecuencia & Porcentaje \\
0 & 66 & 47,14 \\
1 & 31 & 22,14 \\
2 & 29 & 20,71 \\
3 & 5 & 3,57 \\
4 & 9 & 6,43 \\
Total & 140 & 100 \\
\hline
\end{tabular}

(0-1) Bebedor social. (2) Consumo de riesgo. (3) Consumo perjudicial. (4) Dependencia alcohólica.

Para quienes se declararon bebedores se aplicó el cuestionario CAGE, el que clasifica a los bebedores según puntaje obtenido en: bebedores sociales (0-1), consumo de riesgo (2), consumo perjudicial (3) y dependencia alcohólica (4), según el puntaje obtenido (Tabla 2).

Considerando las puntuaciones entre 0-2 como consumo no prejudicial y las puntuaciones 3-4 como consumo perjudicial, $69,3 \%$ se situaron en la primera categoría, versus $30,7 \%$ que se encontraron en la categoría de consumo perjudicial. Del total de hombres bebedores 34,3\% se situaron en la categoría de consumo perjudicial, mientras que 26,9\% de las mujeres bebedoras se ubicó en dicha categoría $(\mathrm{p}=0,344)$. La relación consumo perjudicial de alcohol y curso, no mostró una asociación significativa $(\mathrm{p}=0,158)$.

Es importante destacar que $6,4 \%$ (9 personas) de los bebedores encuestados presentaban depen- dencia alcohólica. Del total de hombres, 5,48\% presentaron dependencia alcohólica $(n=4)$, mientras que $7,46 \%$ de las mujeres $(n=5)$ se clasificaron como dependientes $(\mathrm{p}=0,633)$.

Los bebedores encuestados que se situaron en la categoría de mayor riesgo según CAGE (CAGE 4), presentaron una edad significativamente mayor que los catalogados en menor riesgo (edad promedio $23,1 \pm 2,1$, IC $95 \% 21,5-24,7$ vs 21,4 $\pm 2,0$, IC $95 \% 21,1-21,8, \mathrm{p}=0,0132$ ).

Se analizaron los resultados obtenidos en esta investigación con los proporcionados por un estudio similar realizado en la Pontificia Universidad Católica de Chile el año 2005, a estudiantes de medicina de dicha universidad. La prevalencia de consumo de alcohol en el período de noviembre de 2005 alcanzó 74\% en alumnos de la PUC, mientras que en los estudiantes de medicina de la Universidad Católica del Maule la prevalencia fue de $64,2 \%$, la diferencia porcentual es de $9,8 \%$, IC 0,7-19,3\%, ( $\mathrm{p}=0,0345)$.

Con respecto a la cifra de prevalencia de consumo de alcohol a nivel nacional, según el Octavo Estudio Nacional de Drogas en población general de Chile 2008, realizado por CONACE, el 60,8\% de los jóvenes entre 19 y 25 años de edad consume alcohol por lo menos una vez al mes, cifra menor a la registrada en la muestra de jóvenes de rango etario similar en este estudio.

\section{Discusión}

Una gran cantidad de estudios relacionados al tema señalan que son los jóvenes quienes poseen las cifras más altas en el consumo de alcohol ${ }^{9-10}$. 
Dentro de este contexto los estudiantes universitarios son lo que representan en gran medida a este grupo etario. Sin embargo, no existe mucha literatura que investigue el tema y en cuanto a la subpoblación de estudiantes de medicina, la bibliografía es casi inexistente tanto a nivel internacional como nacional, siendo nuestro estudio el primero a nivel regional ${ }^{11}$.

La prevalencia del consumo de alcohol en los estudiantes involucrados en el estudio es de 64,2\%, cifra similar a la de estadísticas nacionales del CONACE del año 2008, donde se observó que el $60,8 \%$ de los jóvenes de este mismo grupo etario (19 a 25 años de edad) consumía alcohol.

Ahora bien, al comparar nuestros resultados con los proporcionados por un estudio similar realizado en la Pontificia Universidad Católica de Chile el año 2005, a estudiantes de medicina de dicha universidad, se vio que en este grupo de estudiantes la prevalencia de consumo de alcohol en el mes de noviembre de 2005 es de 74\%. Así podemos decir que las cifras de prevalencia del consumo de alcohol del grupo PUC son 15,3\% mayores que las de nuestro grupo de estudio, lo que es estadísticamente significativo $(\mathrm{p}=0,0345)$. La diferencia existente puede ser producida por la ciudad en la que se encuentran las distintas casas de estudio, ya que la vida universitaria es completamente distinta en una capital nacional que en provincia, en el sentido de estímulos estresantes y calidad de vida. Sin embargo, no se puede establecer conclusiones al respecto, porque el presente estudio no evaluó aquellos factores.

Por otra parte, al analizar lo que ocurre dentro de nuestra población, encontramos que existe una asociación estadísticamente significativa entre género y consumo de alcohol, aunque las mujeres se encuentran más representadas que los hombres en nuestra muestra, el número de hombres encuestados es suficiente para establecer conclusiones. Así encontramos que son los hombres quienes presentan una mayor prevalencia de consumo de alcohol, lo que refleja exactamente lo que ocurre a nivel nacional ${ }^{9,12}$. Sin embargo, esta asociación no existe en el estudio realizado por la PUC donde de muestra que el consumo es igual tanto en mujeres como hombres, hecho que también se ha observado en estudios de países desarrollados como Turquía y el Reino Unido ${ }^{7}$. Es posible que la realidad de Santiago de Chile se esté acercando a la tendencia internacional donde el consumo es similar entre hombres y mujeres, pero en provincia esto aún no ocurre, aunque esta tendencia podría cambiar en un futuro próximo, ya que se ha visto que el consumo de alcohol en mujeres ha ido aumentando en los últimos 10 años, mientras que el de los hombres se ha mantenido estable ${ }^{12}$.

En cuanto a consumo y año de carrera se observó que es muy frecuente en los cursos primero y segundo, luego disminuye en los cursos cuarto y quinto y se hace máximo en los estudiantes de internado. Si bien, estas diferencias no son significativas, la tendencia a una distribución en "U”, pudiera existir y no haberse demostrado por una población pequeña en que el error de tipo II es posible y ella y los factores asociados podrían ser sujeto de posteriores estudios. Así mismo es importante destacar que la muestra no es muy representativa de tercero, cuarto y aún menos de internado, sin embargo es muy representativa de primero y segundo. Otra alternativa para esta tendencia sería el posible efecto confusor del género, ya que en el internado la totalidad de los encuestados fueron varones. Si dejáramos fuera a este grupo de internado que presenta una baja representatividad, podríamos observar que la tendencia es a disminuir el consumo de alcohol a medida que se avanza en la carrera, lo que podría estar dando luces que en nuestra casa de estudio, los alumnos podrían estar tomando conciencia de lo que significan los riesgos en el consumo de alcohol a medida que adquieren mayores conocimientos respecto al tema. Ante esta evidencia, se hacen necesarios nuevos estudios dirigidos a analizar las motivaciones del consumo de alcohol. Por otra parte, debe tenerse en cuenta que esta distribución por curso, no se observa en el estudio publicado por la PUC ni tampoco en estudios internacionales, donde se ve que mientras más avanzado en la carrera de medicina, mayor es el consumo de alcohol ${ }^{7,11,13}$.

$\mathrm{Al}$ analizar los resultados CAGE se encontró que $6,43 \%$ de los bebedores encuestados presentan dependencia alcohólica, donde la mayoría eran mujeres, aunque esta diferencia no fue significativa. Esto nos parecen dos datos de alarma, considerando que la dependencia alcohólica constituye una psicopatología que va afectando los distintos sistemas con los que se relaciona la persona, ya sea familiar, social y profesional y que requiere un tratamiento especializado. Se encontró, además, que la edad se asocia al consumo perjudicial, es 
decir que a mayor edad independiente de la prevalencia de consumo, los que sí lo hacen presentan un grado mayor de dependencia, en quienes por consecuencia sería más difícil prevenir y tratar el consumo de alcohol.

Dentro de los desafíos futuros que nacen a partir de este estudio, se encuentra la realización de nuevas investigaciones que validen los resultados encontrados y busquen factores de riesgo asociados, para proyectar intervenciones destinadas a disminuir el consumo de alcohol perjudicial y la dependencia alcohólica, y además, potenciar el consumo de alcohol responsable y moderado, en especial en alumnos de Medicina.

Agradecimientos: $\mathrm{Al}$ grupo de alumnos de $2^{\circ}$ año de medicina que colaboró con la encuesta de este estudio: Oscar Heredia, Catalina López, Carolina Navarrete, Constanza Soto, Manuel Valenzuela.

\section{Referencias}

1. Lieber CS. Medical disorders of alcoholism. N Engl J Med 1995; 33: 1058-65.

2. Global status report on alcohol and health. WHO 2011.

3. CONACE. Octavo estudio nacional de drogas en población general de Chile 2008.

4. ANALES. De la facultad de medicina de la Universidad Nacional Mayor de San Marcos. ISSN 1025-5583 vol. 62,
$\mathrm{N}^{\circ} 12001$.

5. Abreu García MT, Fernández Albán M, Martín García AA. Comportamiento del consumo de bebidas alcohólicas en estudiantes de medicina. Revista Cubana de Salud Pública 1995.

6. Akvardar Y, Demiral Y, Ergor G, Ergor A, Bilici M, Ozer OA. Substance use in a sample of Turkish medical students. Drug Alcohol Depend 2003; 72: 117-21.

7. Romero M, Santander J, Hitschfeld M, Labbé M, Zamora V. Consumo de tabaco y alcohol entre los Estudiantes de Medicina de la Pontificia Universidad Católica de Chile. Rev Med Chile 2009; 137 (3): 361-8.

8. Ewing JA. Detecting Alcoholism. The CAGE questionnaire. JAMA 1984; 252 (14): 1905-7

9. CONACE. Informe sobre uso de drogas en estudiantes de la educación superior. Quinto estudio nacional de drogas en población general de Chile 2002; junio 2003.

10. Sapag J, Florenzano R. Adolescencia: promoción, prevención y atención de salud. Ediciones Universidad Católica 2002; 201-18.

11. Newbury-Birch D, Walshaw D, Kamali F. Drink and drugs: From medical students to doctors. Drug and Alcohol Dependence 2001; 64: 265-70.

12. CONACE. Situación del consumo de drogas en mujeres de Chile. Quinto estudio nacional de drogas en población general de Chile 2002; agosto 2004.

13. Mcauliffe W, Rohman M, Breer P, Wyshak G, Santangelo S, Magnuson E. Alcohol use and abuse in random samples of physicians and medical students. Am J Public Health 1991; 81: 177-82. 